\title{
Telomere Instability in Lynch Syndrome Families Leads to Some Shorter Telomeres in $\mathrm{MSH} 2+$-- Carriers
}

\author{
M. Carmen Garrido-Navas $1,2,3, * \mathbb{1}$, Frances Tippins ${ }^{1}$, Julian Barwell ${ }^{1}$, Jonathan Hoffman ${ }^{4}$, \\ Veryan Codd ${ }^{5}$ and Nicola J. Royle ${ }^{1, * \mathbb{D}}$ \\ 1 Department of Genetics and Genome Biology, University of Leicester, Leicester LE1 7RH, UK; \\ frances.tippins@nhs.net (F.T.); Julian.Barwell@uhl-tr.nhs.uk (J.B.) \\ 2 Liquid Biopsies \& Cancer Interception (LiqBiopCI) Group, Junta de Andalucía de Genómica Investigación \\ Oncológica, GENYO-Centro Pfizer-Universidad de Granada, 18016 Granada, Spain \\ 3 Universidad Internacional de la Rioja, 137, 26006 Logroño, La Rioja, Spain \\ 4 Clinical Genetics Unit, Birmingham Women's Hospital, Birmingham B15 2TG, UK; jonathanhoffman@nhs.net \\ 5 Department of Cardiovascular Sciences, University of Leicester, BHF Cardiovascular Research Centre, \\ Glenfield Hospital, Leicester LE3 9QP, UK; vc15@leicester.ac.uk \\ * Correspondence: carmen.garrido@genyo.es (M.C.G.-N.); njr@leicester.ac.uk (N.J.R.)
}

Received: 21 September 2020; Accepted: 29 October 2020; Published: 31 October 2020

\begin{abstract}
Lynch syndrome (LS) is an inherited predisposition to early onset of various cancers, caused by mutation in a DNA mismatch repair (MMR) gene. In heterozygous $\mathrm{MMR}^{+/-}$carriers, somatic mutation, loss or silencing of the wild type allele increases the mutation rate, facilitating the initiation of MMR-defective cancers. These cancers are characterized by instability at short tandem repeats (STRs) and in telomeric DNA. We have investigated telomere length in saliva DNA from LS and control families, using single telomere analysis at XpYp and 12q and by qPCR to measure total telomeric DNA. Single telomere analysis showed a trend for shorter XpYp telomeres in $\mathrm{MSH}^{+/-}$ carriers compared to $\mathrm{MLH1}^{+/-}$carriers or controls, but this was masked in the comparative analysis of total telomeric DNA. Comparison of age-adjusted telomere length within families showed that neither $\mathrm{MSH}_{2}^{+-}$or $\mathrm{MLH1}^{+-}$children had consistently shorter or longer telomeres than their $\mathrm{MMR}^{+/-}$parent, indicating the absence of an inter-generational effect on telomere length. Unexpectedly however, wildtype children in families with MSH2 mutations, had significantly longer XpYp telomeres than their $\mathrm{MMR}^{+/-}$parent. Altogether our data suggest that MMR insufficiency, particularly in $\mathrm{MSH}^{+/-}$ carriers, increases telomere instability and somatic cell turnover during the lifetime of LS mutation carriers but has minimal consequences for telomere length in the germline.
\end{abstract}

Keywords: telomeres; DNA mismatch repair; $M S H 2$; $M L H 1$; Lynch syndrome

\section{Introduction}

Telomeres are the essential capping structures of chromosomes with well-established roles in ageing and cancer. Short telomeres activate a DNA damage response and cells with one or a few dysfunctional telomeres enter a senescent state. Cancer cells usually acquire mutations that allow them to bypass this replication barrier but must eventually activate a telomere maintenance mechanism [1-3]. Inheritance of a mutant allele at a DNA mismatch repair (MMR) gene causes Lynch syndrome (LS), a predisposition to early-onset of a variety of cancers with microsatellite instability [4]. Heterozygous carriers of $\mathrm{MLH1}$ or MSH2 mutations show a higher level of instability at short tandem repeat (STR) loci (also known as microsatellite instability, MSI), with a bias towards deletions, than seen in their non-carrier relatives. This shows that heterozygous $\mathrm{MMR}^{+/-}$cells have a limited capacity for MMR arising from haplo-insufficiency [5-7]. 
Telomeric DNA comprises tandem arrays of (TTAGGG) $)_{n}$ often interspersed with sequence-variant repeats at the proximal end of the telomere [8]. Thus telomeric DNA shares some features with STRs that, as stated, are unstable in MMR deficient cells primarily due to slippage on the repetitive DNA during replication [9]. There are additional obstacles that cause the replication fork slow or stall on telomeric DNA during S-phase. These include G-quadruplex structures that form on the (TTAGGG) strand and the requirement to unwind the t-loop capping structure. TRF1, an essential component of shelterin, is required for the efficient replication of telomeric DNA as it facilitates G-quadruplexes removal and prevents the manifestation of fragile telomeres [10]. We have shown that telomeric DNA is unstable in LS colorectal cancers (CRCs) and tumor derived cell lines that lack a functional MSH2 gene, resulting in frequent gains and losses of telomeric repeats [11,12]. Telomeres also shortened faster in a primary human cell line with depleted MSH2 protein [13]. It is thought that the instability of telomeric DNA in MMR defective cells is associated with replication slippage, but whether this is also influenced by G-quadruplex formation and replication fork stalling is currently unknown.

It has been proposed that LS families show genetic anticipation, with the first cancer appearing at an earlier age in successive generations. This hypothesis is controversial [14] with some studies showing evidence that supports genetic anticipation, in particular in families with MSH2 mutations, while data from other studies indicating that such observations may be explained by other factors $[15,16]$. The controversy persists partly because there is inadequate evidence for a biological mechanism that could cause genetic anticipation in LS [14]. Genetic anticipation, associated with inter-generational telomere shortening, is seen in various forms of dyskeratosis congenita (DC), for example in families with autosomal dominant DC caused by mutations in the telomerase RNA component (TERC) [17], and other short telomere disorders [18]. However, it is not known whether a subtle effect on telomeric DNA, associated with MMR deficiency, could contribute to genetic anticipation in LS [19]. In this study we investigated whether $\mathrm{MSH}_{2}{ }^{+/-}$and $\mathrm{MLH1}^{+/-}$carrier status affects telomere length within LS families. We did not find evidence of an inter-generational effect on telomere length between $\mathrm{MMR}^{+/-}$parents and children. In contrast, single telomere length analysis (STELA) showed that wild type (WT) children in LS families tended to have some longer telomeres than their $\mathrm{MMR}^{+/-}$parent and siblings, particularly when $M S H 2$ was mutated. Overall the data support the hypothesis that MMR insufficiency, particularly when MSH2 is mutated, increases telomere instability and subsequent cell turnover in somatic tissues but has little consequence for telomere length in the germline where telomerase is active.

\section{Materials and Methods}

Saliva samples were collected from adult individuals (18 years or older) in control and LS families. All subjects gave their informed consent for inclusion before they participated in the study. The study was conducted in accordance with the Declaration of Helsinki. Control families $(n=10)$, comprised 37 individuals with a mean age $45.3 \pm 18.4$ (range 18.2 to 82.6 years) and were recruited with approval from the University of Leicester's Research Ethics Committee (Ref: njr-61d3). Lynch syndrome families $(n=24)$ comprising 91 individuals with a mean age $47.0 \pm 15.8$ (range 19.2 to 73.1 years) were recruited in the Birmingham Women and Children's Hospital (BWH) and the Leicester Royal Infirmary (LRI) under the ethical approval for the 'Molecular Pathology of Human Genetic Disease (HumGenDis)' study (REC reference CA/5175; and UHL10942/ IRAS 50895). Saliva samples were collected from two generations in each family with the older generation being designated as 'parents' and the younger generation as 'children'. The one exception was the BWHF105 family, with one saliva sample also collect form a third generation. In this family, the individual BWHF105 G4P9 is the child in one comparison and the parent in another.

\subsection{Sample Processing and Telomere Length Measurements}

DNA was extracted from saliva samples using a DNA saliva kit (Oragene, Genotek, Ottawa, ON, Canada). The samples were coded and telomere length analyses conducted blind. STELA was 
used to amplify XpYp and 12q telomeres as described previously [20]. The analysis of individual chromosomes ends using STELA facilitates the detection of subtle effects on telomeres, for example the frequency of very short dysfunctional telomeres that might trigger cell senescence or subtle changes to length. The XpYp and 12q telomeres were selected because there is substantial evidence that the primers used in STELA are specific to these chromosomes ends. Primers designed to some other telomere-adjacent regions amplify from an unknown number of chromosome ends, which may also vary between individuals. Telomere length measurement using STELA from an unknown number of chromosome ends could complicate the comparative analysis in an unpredictable manner. Furthermore, we previously demonstrated that XpYp and 12q telomeres become unstable in MMR deficient cell lines and shorten at a faster rate than MMR-proficient cells [13]. In addition, XpYp STELA products from a lymphoblastoid cell line (KK) were included in all gels as a control for length analysis. Total abundance of telomeric repeats (T) was assessed by quantitative PCR (qPCR) using amplification of a single copy gene (S, RPLP0 (aka 36B4)) as a reference to report the T/S ratio for each sample [21]. The inter-run coefficient of variation for the qPCR measurements was $3.54 \%$.

\subsection{Statistical Analyses}

Linear regression and other statistical analyses were conducted using Graph-Pad Prism, version 7.0. Age-adjusted telomere lengths ( $\Delta \mathrm{Tel})$ were calculated as described previously [17] and child/parent comparisons of the $\Delta$ Tel age-adjusted values calculated. Distributions of $\Delta$ Tel child- $\Delta$ Tel parent values were compared using the non-parametric Mann-Whitney and Kruskal Wallis tests for two or more groups, respectively.

\section{Results}

High molecular weight DNA was extracted from the saliva samples collected from the control and LS families described in Table 1 and Figure S1.

\subsection{Cross-sectional Analysis of Total Telomeric DNA and Individual Telomere Lengths in LS and Controls}

Median telomere lengths were measured in saliva DNA from control and LS families using STELA at XpYp and 12q (Figure 1) and total telomeric DNA abundance was assessed by T/S-qPCR.

In the control cohort, all three telomere measurements exhibited the expected decrease with age, and linear regression analyses showed slopes that were significantly different from zero (Figure 2a, Table S1). These cross-sectional analyses are consistent with the XpYp and 12q telomeres shortening at an average $21.8 \pm 7.6$ and $16.7 \pm 5.6$ bp per year, respectively, in the control cohort. The T/S-qPCR data from LS carriers $\left(\mathrm{MMR}^{+/-}\right)$also showed a significant decline with age. In contrast, the graphs of median XpYp and 12q telomere lengths versus age in the LS cohort showed lower $\mathrm{r}^{2}$ and $\mathrm{r}$ values and the slopes were not significantly different from zero (Figure 2a, Table S1). This suggests that the length dynamics at individual telomeres are different in $\mathrm{MMR}^{+/-}$carriers, a feature that is masked in the qPCR data. Nevertheless, the linear regression slopes, which represent the cross-sectional age-related shortening, were not significantly different between the control and $\mathrm{MMR}^{+/-}$cohorts (Table S1). To determine whether MLH1 and MSH2 mutations are associated with different effects on telomere length, regression analyses were conducted with the $\mathrm{MMR}^{+/-}$cohort split by genotype. This also revealed very weak negative correlations between age and XpYp and 12q telomere lengths that were not significantly different from zero (Figure 2b; Table S1). The $\mathrm{MSH}^{+/-}$mutation carriers tended to have shorter XpYp telomeres than the $M H L 1^{+-}$carriers (Figure 2b), with a lower Y-intercept $(p=0.0427$, Table S1), suggesting that $\mathrm{MSH}^{+-}{ }^{+-}$individuals start adulthood with some shorter telomeres. 
Table 1. Description of Lynch syndrome (LS) and control families.

\begin{tabular}{|c|c|c|c|c|c|c|c|c|}
\hline & \multicolumn{4}{|c|}{ LS Families $(\mathrm{N}=24)$} & \multicolumn{4}{|c|}{ Control Families $(\mathbf{N}=10)$} \\
\hline & \multicolumn{2}{|c|}{$\begin{array}{l}\text { MMR Mutation-Carriers } \\
\qquad(\mathrm{N}=60)\end{array}$} & \multicolumn{2}{|c|}{$\begin{array}{l}\text { Non-Carriers } \\
\quad(\mathbf{N}=31)\end{array}$} & \multirow{3}{*}{$\begin{array}{c}\text { Total } \\
91\end{array}$} & \multicolumn{2}{|c|}{$\begin{array}{l}\text { Controls } \\
(\mathrm{N}=37)\end{array}$} & \multirow{3}{*}{$\begin{array}{c}\text { Total } \\
37\end{array}$} \\
\hline & Cancer & Cancer-free & Cancer & Cancer-free & & Cancer & Cancer-free & \\
\hline $\mathrm{n}$ & 32 & 28 & 2 & 29 & & 3 & 34 & \\
\hline Parent: N (\%) & $24(75)$ & $\mathrm{b}_{3}(11)$ & $2(100)$ & c $15(52)$ & $44(48)$ & $3(100)$ & $15(44)$ & $18(49)$ \\
\hline Children: $\mathrm{N}(\%)$ & $8(25)$ & $25(89)$ & $0(0)$ & $14(48)$ & $47(52)$ & $0(0)$ & $19(56)$ & $19(51)$ \\
\hline $\mathrm{MSH}^{+/-}: \mathrm{N}(\%)$ & $15(47)$ & $18(64)$ & - & - & $33(36)$ & - & - & \\
\hline $\mathrm{MLH}^{+/-}: \mathrm{N}(\%)$ & $17(53)$ & $10(36)$ & - & - & $27(30)$ & - & - & \\
\hline${ }^{\mathrm{a}} \mathrm{MMR}^{+/+}: \mathrm{N}(\%)$ & - & - & $2(100)$ & $29(100)$ & $31(34)$ & $3(100)$ & $34(100)$ & $37(100)$ \\
\hline \multirow[t]{2}{*}{ Sex: N (\%) } & F: $14(44)$ & F: $17(61)$ & F: $2(100)$ & F: $16(55)$ & F: $49(54)$ & F: $2(67)$ & F: $21(62)$ & $23(62)$ \\
\hline & M: 18 (56) & M: 11 (39) & M: $0(0)$ & M: 13 (45) & M: $42(46)$ & M: 1 (33) & M: $13(38)$ & $14(38)$ \\
\hline Mean age at sample \pm SD parents & $60 \pm 8$ & $66 \pm 2$ & $65 \pm 11$ & $63 \pm 7$ & $62 \pm 8$ & $56 \pm 3$ & $62 \pm 10$ & $61 \pm 10$ \\
\hline Mean age at sample \pm SD children & $35 \pm 8$ & $33 \pm 7$ & - & $35 \pm 8$ & $34 \pm 7$ & - & $31 \pm 11$ & $31 \pm 11$ \\
\hline Mean age 1 st cancer \pm SD parents & $45 \pm 11$ & - & $59 \pm 9$ & - & - & $44 \pm 8$ & - & - \\
\hline Mean age 1st cancer \pm SD children & $24 \pm 7$ & - & - & - & - & - & - & - \\
\hline
\end{tabular}

Abbreviations: $\mathrm{n}$ : number of families or individuals; $N(\%)$ : number of subjects and percentage within a group; ${ }^{a} \mathrm{MMR}^{+/+}$: homozygous for a wild type MMR gene; F: female; M: male. SD: standard deviation; ${ }^{\mathrm{b}}$ One $\mathrm{MSH}^{+/-}$individual in a three generation LS family was treated as child and as parent. ${ }^{\mathrm{c}} 15 \mathrm{MMR}^{+/+}$parents in the LS families were included as controls in age versus telomere length graphs (total controls $=52$ ). 

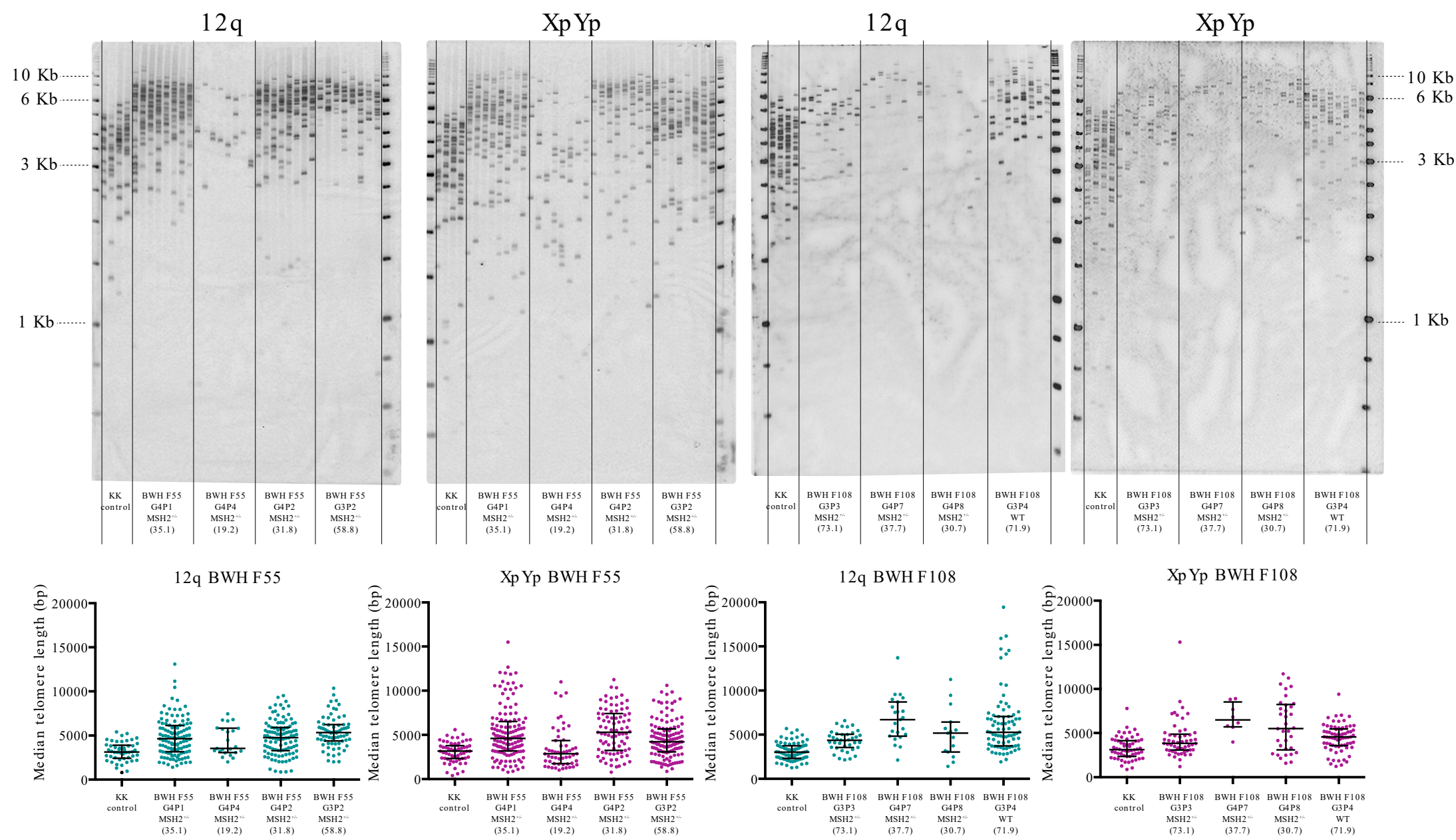

Figure 1. Examples of XpYp and 12q single telomere length analysis (STELA) in two LS families. Top panel, STELA southern blots for 12q and XpYp telomeres in four individuals from two families (BWH F55 and BWH108). XpYp STELA products from the KK cell line were included as a control. Bottom panel, scatter plots for quantification of median telomere length. Unique codes for each individual identify generation $(\mathrm{G})$ and position in the pedigree (P). Numbers in brackets indicate the age (in years) at sample collection. 
a

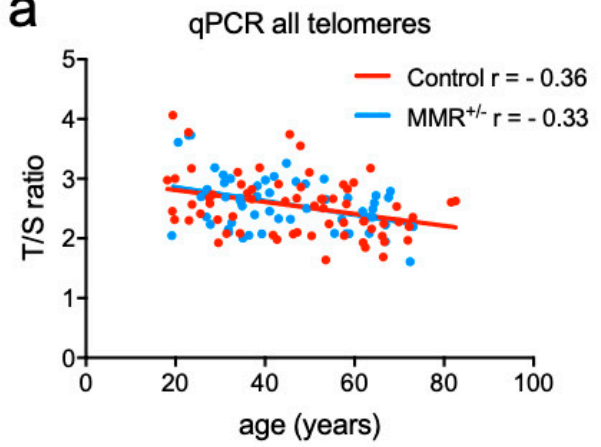

b

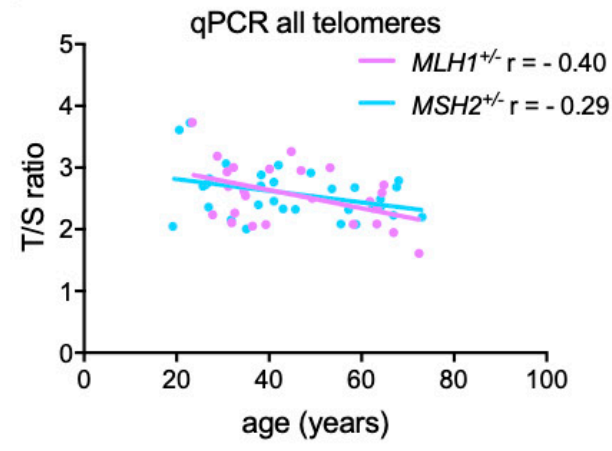

XpYp telomere

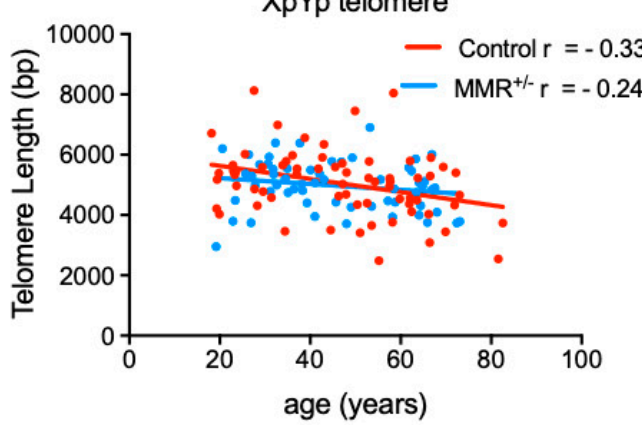

age (years)

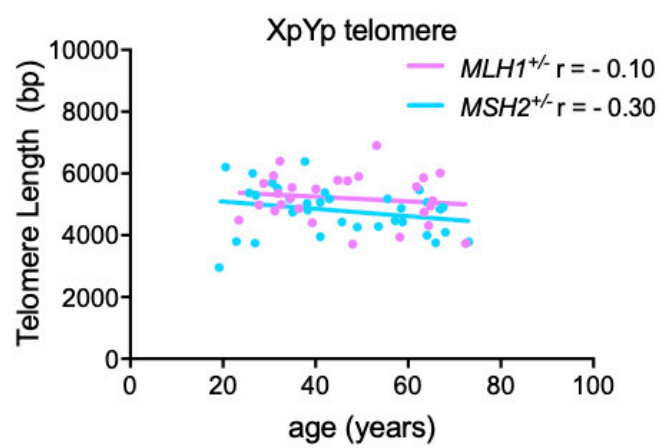

12q telomere
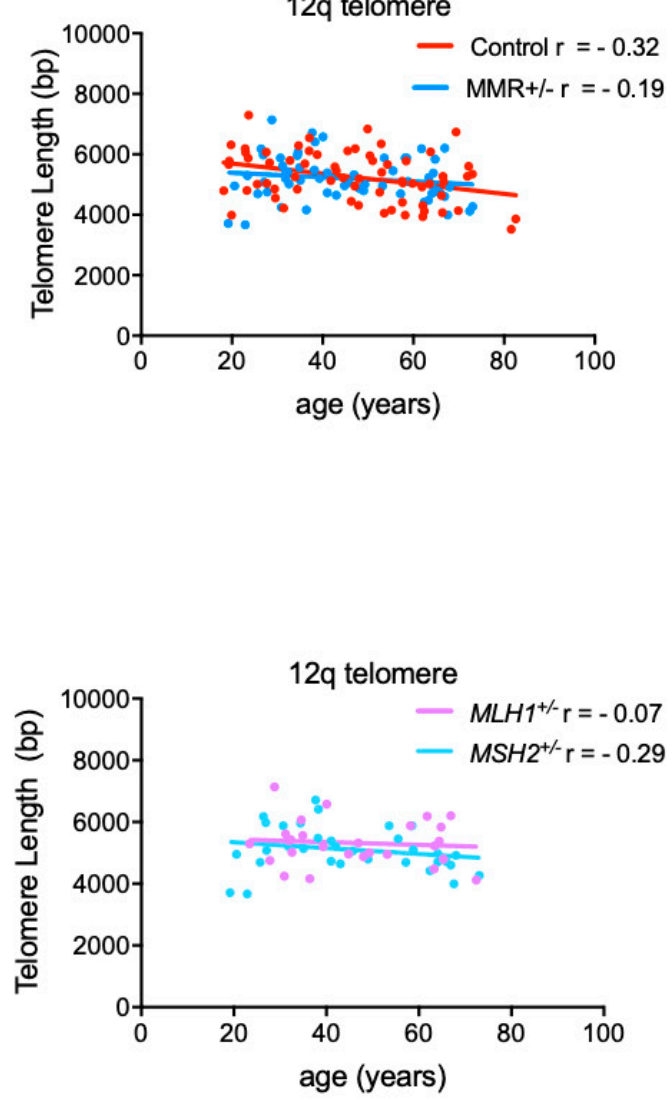

Figure 2. Graphs of telomere measurements versus age for control and Lynch syndrome cohorts. All graphs show telomere length measurements (Y axis) plotted against age (X axis) for each individual. (a) Shows the linear regression analysis for LS (MMR $\left.{ }^{+/-}\right)$in blue $(n=60)$ and healthy controls in red $(n=68)$ for all (T/S-qPCR) telomeres, XpYp and 12q telomeres. (b) Shows comparison of the linear regression lines for $M H S 2^{+/-}$in light blue $(n=33)$ and $M L H 1^{+/-}$in mauve $(n=27)$ for all telomeres, $\mathrm{XpYp}$ and 12q telomeres. Spearman correlation values (r) are shown for all graphs. 


\subsection{Comparison of Age-adjusted of Telomere Lengths within Families}

To investigate inter-generational differences in telomere length in LS and control families, telomere measurements were compared after adjusting for age ( $\Delta \mathrm{Tel})$ [17]. The difference in the aged-adjusted telomere measurements between a child and a parent $\left(\Delta \mathrm{Tel}_{\text {child }}-\Delta \mathrm{Tel}_{\text {parent }}\right.$, Table S2), showed that the $\mathrm{MMR}^{+/-}$children did not have consistently shorter telomeres than either WT or MMR ${ }^{+/-}$parent. In contrast, the WT children in LS families had significantly longer age-adjusted XpYp telomeres than their $\mathrm{MMR}^{+/-}$parent (Figure 3a). A similar non-significant trend was seen at the 12q telomere but not in the T/S-qPCR data. Subdivision of the $\mathrm{MMR}^{+/-}$families according to gene mutation $\left(\mathrm{MLH1}^{+/-}\right.$or $\mathrm{MSH}^{+/-}$) reduced the number of child-parent comparisons that could be made, but it confirmed that WT and not $\mathrm{MSH}^{+/-}$or $\mathrm{MLH1}^{+/-}$children tend to have longer XpYp telomeres than their $\mathrm{MSH}^{+/-}$or $\mathrm{MLH1}^{+/-}$parent (Figure 3b).
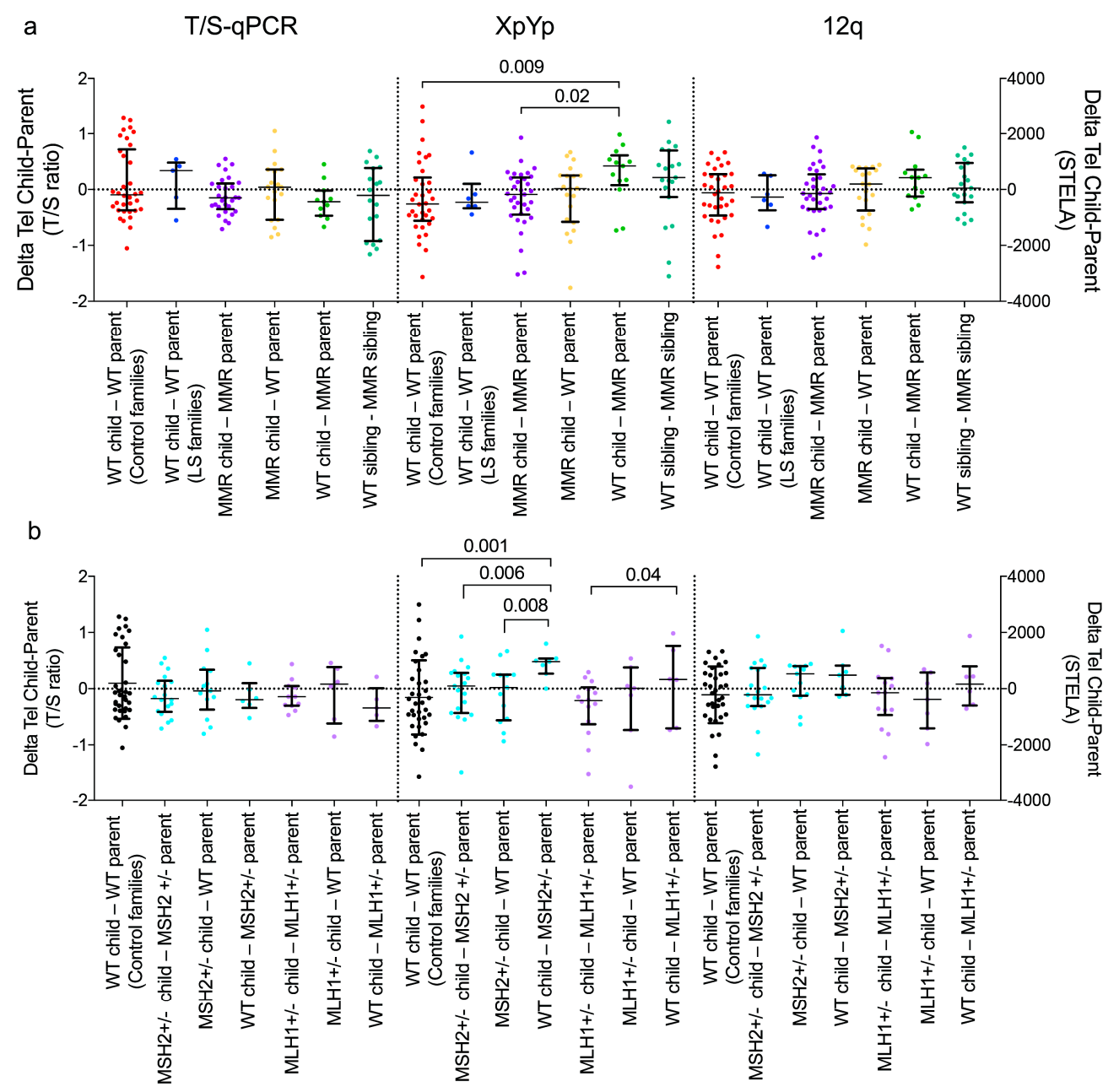

Figure 3. Comparison of age-adjusted telomere measurements within families. (a) Scatter plots of the difference between age-adjusted telomere measurements $(\Delta \mathrm{Tel})$ for children and parents in the control and LS families and between WT and MMR+/- siblings in the LS families. The analysis is shown for the T/S-qPCR, XpYp and 12q data with median and interquartile ranges. (b) Scatter plots are shown for LS families with MSH2 or MLH1 mutations. The scatter plot distributions were compared using a non-parametric Kruskal Wallis test.

\section{Discussion}

The aim of this study was to determine whether haplo-insufficiency for DNA MMR impacts on telomere length in heterozygous carriers of MMR gene mutations. The various methods for telomere length measurement each have advantages and disadvantages [22]. Therefore, we used complementary 
approaches including the T/S-qPCR method to assess total abundance of (TTAGGG) DNA and STELA to measure median lengths at the XpYp and 12q telomeres in saliva DNA samples from control and LS families [20,21].

The cross-sectional analyses of XpYp and 12q telomere lengths in the healthy controls showed significant shortening with age, in line with other studies [23,24]. In contrast, the same analysis in $\mathrm{MMR}^{+/-}$carriers did not show a strong negative correlation between telomere length and age. This may suggest that there is a wider distribution of telomere lengths at any age among $\mathrm{MMR}^{+/-}$carriers, which could be a consequence of $\mathrm{MMR}^{+/-}$driven telomere instability. In addition, the cross-sectional and Y-intercept analyses showed that adult $\mathrm{MSH}^{+/-}$carriers tended to have shorter XpYp telomeres, than controls and $\mathrm{MLH1}^{+/-}$carriers. MSH2 is involved, as a heterodimer with MSH6 or MSH3, in the detection and binding to mismatches or insertion/deletion loops in DNA but also in the role that the MMR machinery plays in the cell cycle response to DNA damage. The data presented raise the possibility that MSH2 deficiency has a greater effect on telomere dynamics than MLH1 deficiency but the consequences may vary between telomeres. The question then arises as to why some telomeres might be more adversely affected by MSH2 deficiency than others. One possibility is variation in the composition and density of telomere variant repeats (TVRs) in the proximal regions of human telomeres, as this is known to vary considerably between chromosome ends, alleles and populations $[8,12,25,26]$. Differences in the extent of TVR composition between the XpYp and 12q telomeres is likely to influence the dynamics of telomere repeat turnover $[8,12,26]$, as indicated in a comparative analysis of TVR abundance in LS and sporadic colorectal cancers [27].

The subtle differences we identified between individual telomeres indicate that telomere length dynamics differ between controls and MMR mutation carriers, particularly if $\mathrm{MSH} 2$ is mutated but these differences were masked in the bulk analysis of all telomeres (using T/S-qPCR, Figure 2 and Table S1). STELA has the advantage that it detects short, potentially dysfunctional telomeres efficiently, but it is limited to the analysis of only a few telomeres, whereas T/S-qPCR measures mean (TTAGGG) abundance from all telomeres, but may under-represent very short or very long telomeres [28]. In this study, the intra-individual comparison of the XpYp and 12q telomere lengths measured by STELA, showed a stronger positive correlation than between individual telomere length measurements and the T/S-qPCR value (Figure S2), which probably reflects the different advantages and disadvantages of the methods.

For MMR haplo-insufficiency to contribute to genetic anticipation in LS families we proposed that $\mathrm{MMR}^{+/-}$children would show shorter age-adjusted telomere length than their $\mathrm{MMR}^{+/-}$parents. In fact, comparison of parent-child age-adjusted telomere lengths showed that $\mathrm{MMR}^{+/-}$children did not have significantly shorter or longer telomeres than either parent. Consequently, we did not find evidence of an inter-generational effect on telomere length that might be attributed to shorter telomere length in gametes. In contrast, WT children had significantly longer age-adjusted XpYp telomeres than their $\mathrm{MMR}^{+/-}$parents, with a stronger effect in $\mathrm{MSH} 2$ families, and a similar non-significant trend at 12q. Moreover, $\mathrm{MSH}^{+/-}$carriers tend to start adulthood with shorter telomeres than WT individuals. Altogether the data presented here show there is a complex relationship between telomere instability and turnover of somatic cells during the lifetime of $\mathrm{MMR}^{+/-}$carriers, in particular $\mathrm{MSH}^{+/-}$individuals.

Supplementary Materials: The following are available online at http://www.mdpi.com/2075-1729/10/11/265/s1, Figure S1: Pedigrees of families analyzed; Figure S2: Comparison of telomere length measurement methods; Table S1: Statistics associated with the cross-sectional analyses of telomere lengths in Figure 2; Table S2: Age-adjusted telomere measurements and comparisons between family members.

Author Contributions: M.C.G.-N., F.T., J.B. and J.H. collected the samples in accordance with the ethical permissions; M.C.G.-N. and F.T. generated and analyzed the STELA data; V.C. conducted the T/SqPCR analysis; N.J.R. initiated the project, obtained the funding and wrote the paper with M.C.G.-N. All authors have read and agreed to the published version of the manuscript.

Funding: M.C.G.-N. was funded by a PhD studentship form the University of Leicester; additional development funding was from the Cancer Research UK Leicester Centre (2014-17). 
Acknowledgments: We would like to thank all patients and healthy donors for their participation in this project. We thank Eamonn Maher most sincerely for his support in obtaining saliva sample from LS families. We would also like to thank Maria Viskaduraki for her advice on the statistical analyses.

Conflicts of Interest: The authors declare no conflict of interest.

\section{References}

1. Stanley, S.E.; Armanios, M. The short and long telomere syndromes: Paired paradigms for molecular medicine. Curr. Opin. Genet. Dev. 2015, 33, 1-9. [CrossRef] [PubMed]

2. Opresko, P.L.; Shay, J.W. Telomere-associated aging disorders. Ageing Res. Rev. 2017, 33, 52-66. [CrossRef] [PubMed]

3. Maciejowski, J.; de Lange, T. Telomeres in cancer: Tumour suppression and genome instability. Nat. Rev. Mol. Cell Biol. 2017, 18, 175-186. [CrossRef] [PubMed]

4. Lynch, H.T.; Lynch, P.M.; Lanspa, S.J.; Snyder, C.L.; Lynch, J.F.; Boland, C.R. Review of the Lynch syndrome: History, molecular genetics, screening, differential diagnosis, and medicolegal ramifications. Clin. Genet. 2009, 76, 1-18. [CrossRef] [PubMed]

5. Alazzouzi, H.; Domingo, E.; Gonzalez, S.; Blanco, I.; Armengol, M.; Espin, E.; Plaja, A.; Schwartz, S.; Capella, G.; Schwartz, S., Jr. Low levels of microsatellite instability characterize MLH1 and MSH2 HNPCC carriers before tumor diagnosis. Hum. Mol. Genet. 2005, 14, 235-239. [CrossRef] [PubMed]

6. Coolbaugh-Murphy, M.I.; Xu, J.P.; Ramagli, L.S.; Ramagli, B.C.; Brown, B.W.; Lynch, P.M.; Hamilton, S.R.; Frazier, M.L.; Siciliano, M.J. Microsatellite instability in the peripheral blood leukocytes of HNPCC patients. Hum. Mutat. 2010, 31, 317-324. [CrossRef]

7. Kansikas, M.; Kasela, M.; Kantelinen, J.; Nystrom, M. Assessing how reduced expression levels of the mismatch repair genes MLH1, MSH2, and MSH6 affect repair efficiency. Hum. Mutat. 2014, 35, 1123-1127. [CrossRef] [PubMed]

8. Baird, D.M.; Jeffreys, A.J.; Royle, N.J. Mechanisms underlying telomere repeat turnover, revealed by hypervariable variant repeat distribution patterns in the human Xp/Yp telomere. EMBO J. 1995, 14, 5433-5443. [CrossRef]

9. Ellegren, H. Microsatellite mutations in the germline: Implications for evolutionary inference. Trends Genet. 2000, 16, 551-558. [CrossRef]

10. de Lange, T. Shelterin-Mediated Telomere Protection. Annu. Rev. Genet. 2018, 52, 223-247. [CrossRef]

11. Pickett, H.A.; Baird, D.M.; Hoff-Olsen, P.; Meling, G.I.; Rognum, T.O.; Shaw, J.; West, K.P.; Royle, N.J. Telomere instability detected in sporadic colon cancers, some showing mutations in a mismatch repair gene. Oncogene 2004, 23, 3434-3443. [CrossRef]

12. Mendez-Bermudez, A.; Hills, M.; Pickett, H.A.; Phan, A.T.; Mergny, J.L.; Riou, J.F.; Royle, N.J. Human telomeres that contain (CTAGGG)n repeats show replication dependent instability in somatic cells and the male germline. Nucleic Acids Res. 2009, 37, 6225-6238. [CrossRef]

13. Mendez-Bermudez, A.; Royle, N.J. Deficiency in DNA mismatch repair increases the rate of telomere shortening in normal human cells. Hum. Mutat. 2011, 32, 939-946. [CrossRef]

14. Ponti, G.; Ruini, C.; Tomasi, A. Mismatch repair gene deficiency and genetic anticipation in Lynch syndrome: Myth or reality? Dis. Colon Rectum 2015, 58, 141-142. [CrossRef] [PubMed]

15. von Salome, J.; Boonstra, P.S.; Karimi, M.; Silander, G.; Stenmark-Askmalm, M.; Gebre-Medhin, S.; Aravidis, C.; Nilbert, M.; Lindblom, A.; Lagerstedt-Robinson, K. Genetic anticipation in Swedish Lynch syndrome families. PLoS Genet. 2017, 13, e1007012. [CrossRef] [PubMed]

16. Ten Broeke, S.W.; Rodriguez-Girondo, M.; Suerink, M.; Aretz, S.; Bernstein, I.; Capella, G.; Engel, C.; Gomez-Garcia, E.B.; van Hest, L.P.; von Knebel Doeberitz, M.; et al. The Apparent Genetic Anticipation in PMS2-Associated Lynch Syndrome Families Is Explained by Birth-cohort Effect. Cancer Epidemiol. Biomark. Prev. 2019, 28, 1010-1014. [CrossRef] [PubMed]

17. Vulliamy, T.; Marrone, A.; Szydlo, R.; Walne, A.; Mason, P.J.; Dokal, I. Disease anticipation is associated with progressive telomere shortening in families with dyskeratosis congenita due to mutations in TERC. Nat. Genet. 2004, 36, 447-449. [CrossRef]

18. Holohan, B.; Wright, W.E.; Shay, J.W. Cell biology of disease: Telomeropathies: An emerging spectrum disorder. J. Cell Biol. 2014, 205, 289-299. [CrossRef] 
19. Segui, N.; Pineda, M.; Guino, E.; Borras, E.; Navarro, M.; Bellido, F.; Moreno, V.; Lazaro, C.; Blanco, I.; Capella, G.; et al. Telomere length and genetic anticipation in Lynch syndrome. PLoS ONE 2013, 8, e61286. [CrossRef]

20. Jeyapalan, J.N.; Mendez-Bermudez, A.; Zaffaroni, N.; Dubrova, Y.E.; Royle, N.J. Evidence for alternative lengthening of telomeres in liposarcomas in the absence of ALT-associated PML bodies. Int. J. Cancer 2008, 122, 2414-2421. [CrossRef]

21. Codd, V.; Nelson, C.P.; Albrecht, E.; Mangino, M.; Deelen, J.; Buxton, J.L.; Hottenga, J.J.; Fischer, K.; Esko, T.; Surakka, I.; et al. Identification of seven loci affecting mean telomere length and their association with disease. Nat. Genet. 2013, 45, 422-427. [CrossRef]

22. Lai, T.P.; Wright, W.E.; Shay, J.W. Comparison of telomere length measurement methods. Philos. Trans. R. Soc. Lond. B Biol. Sci. 2018, 373. [CrossRef] [PubMed]

23. Chen, W.; Kimura, M.; Kim, S.; Cao, X.; Srinivasan, S.R.; Berenson, G.S.; Kark, J.D.; Aviv, A. Longitudinal versus cross-sectional evaluations of leukocyte telomere length dynamics: Age-dependent telomere shortening is the rule. J. Gerontol. A Biol. Sci. Med. Sci. 2011, 66, 312-319. [CrossRef] [PubMed]

24. Daniali, L.; Benetos, A.; Susser, E.; Kark, J.D.; Labat, C.; Kimura, M.; Desai, K.; Granick, M.; Aviv, A. Telomeres shorten at equivalent rates in somatic tissues of adults. Nat. Commun. 2013, 4, 1597. [CrossRef] [PubMed]

25. Coleman, J.; Baird, D.M.; Royle, N.J. The plasticity of human telomeres demonstrated by a hypervariable telomere repeat array that is located on some copies of 16p and 16q. Hum. Mol. Genet. 1999, 8, 1637-1646. [CrossRef]

26. Baird, D.M.; Coleman, J.; Rosser, Z.H.; Royle, N.J. High levels of sequence polymorphism and linkage disequilibrium at the telomere of 12q: Implications for telomere biology and human evolution. Am. J. Hum. Genet. 2000, 66, 235-250. [CrossRef]

27. Nersisyan, L.; Hopp, L.; Loeffler-Wirth, H.; Galle, J.; Loeffler, M.; Arakelyan, A.; Binder, H. Telomere Length Maintenance and Its Transcriptional Regulation in Lynch Syndrome and Sporadic Colorectal Carcinoma. Front. Oncol. 2019, 9, 1172. [CrossRef]

28. Aubert, G.; Hills, M.; Lansdorp, P.M. Telomere length measurement-caveats and a critical assessment of the available technologies and tools. Mutat. Res. 2012, 730, 59-67. [CrossRef]

Publisher's Note: MDPI stays neutral with regard to jurisdictional claims in published maps and institutional affiliations.

(C) 2020 by the authors. Licensee MDPI, Basel, Switzerland. This article is an open access article distributed under the terms and conditions of the Creative Commons Attribution (CC BY) license (http://creativecommons.org/licenses/by/4.0/). 